La population suisse n'a encore jamais joui d'une aussi bonne santé et d'une espérance de vie aussi élevée qu'aujourd'hui. Malgré cette évolution sociétale générale, l'inégalité sociale ne cesse de s'accentuer. Dans ce contexte, il est du devoir de la santé publique d'intervenir en faveur de la création de conditions-cadres sociales, environnementales et médicales permettant à chacun de vivre en bonne santé. Nous saluons l'association des sciences sociales et de la prise en charge médicale à l'occasion de la Swiss Public Health Conference 2018. Nous nous réjouissons de vous accueillir nombreux à cette occasion et de participer à des débats intéressants à la table ronde de la FMH.

Dr méd. Carlos Beat Quinto, membre du Comité central de la FMH, responsable du département Santé publique et professions de la santé

\title{
Better Health Faster: les sciences sociales au service de la santé
}

\author{
Julia Dratva \\ Dr méd., MD MPH, Présidente de la Société suisse des médecins spécialistes en prévention et santé publique
}

Tout doit aller très vite aujourd'hui, mais dans certains cas ce n'est pas assez vite!

On pourrait croire que les inégalités en matière de santé datent d'hier. Tous les médecins en ont entendu parler au cours de leurs études, la sociologie médicale et la recherche en santé publique offrent diverses explications et pourtant, elles existent encore, en Suisse également. La santé, individuelle et collective, repose sur différents facteurs, dont le patrimoine génétique, le comportement, le niveau de formation, les conditions environnementales et de vie ainsi que l'accès au système de soins. En tant que médecins, nous sommes chaque jour confrontés aux conséquences des inégalités en matière de santé.

Bien que le système de soins ne contribue que de l'ordre de 15 à $20 \%$ à l'état de santé de la population, la quasi-totalité des dépenses dites de santé sont consacrées à ce domaine et en particulier à la prise en charge des malades. La progression des maladies chroniques, l'évolution démographique et les possibilités de traitement souvent coûteuses engendrent une augmentation constante des coûts de la santé (72\% depuis 1996) et, encore plus marquée, des primes d'assurance-maladie (107\% depuis 1996). En Suisse, ces dépenses supplémentaires pèsent lourd dans le budget de nombreuses personnes, et notamment de familles. Par conséquent, ce sont les coûts du système de santé et non pas ses bénéfices qui dominent dans le débat public sur la politique de santé. Dans un souci de réduire les coûts à court terme, on cherche souvent à réaliser des économies dans les domaines de la promotion et la préservation de la santé ainsi que de la prévention. Pourtant, ce sont justement ces domaines qui pourraient contribuer à une réduction des coûts à long terme - la prévention du tabagisme, y compris la prévention structurelle, génère l'un des meilleurs retours sur investissement.

\section{La conférence cherchera à mieux com- prendre la manière dont les sciences sociales peuvent contribuer à encourager une politique orientée vers la santé.}

Tout comme la sociologie médicale, les sciences sociales appréhendent la santé individuelle et collective d'une manière qui dépasse le cadre biomédical. Elles nous aident à comprendre comment l'environnement social, les conditions de vie et notre comportement influencent notre santé. Sur la base de ces connaissances, il est possible de développer des moyens d'action en santé publique qui sont complémentaires avec notre système de soins et qui pourraient contribuer à réduire, à court ou à long terme, les inégalités en matière de santé. Ces connaissances sont d'une grande utilité pour les médecins. Elles augmentent non seulement notre compréhension de la santé et des maladies, mais elles aident également à expliquer des tendances et des modifications, par exemple en ce qui concerne l'incidence des maladies ou la fréquentation accrue des services d'urgence; à moyen et à long terme, elles influeront sur le quotidien clinique. C'est pourquoi la SSSP recommande vivement à tous les collègues, membres ou non, de participer à cette conférence.

La première partie de la conférence donnera l'occasion de partager les connaissances actuelles relatives aux facteurs déterminants de la santé. Il sera question des 
Correspondance: ZHAW, Departement Gesundheit, Institut für Gesundheitswissenschaften Technikumstrasse 71 Postfach

CH-8401 Winterthur Tél. +41589346372 julia.dratva[at]zhaw.ch objectifs de développement durable inscrits dans l'agenda 2030 et de leur incidence sur la politique extérieure suisse en matière de santé, mais également de notre comportement individuel. La deuxième partie sera consacrée à l'analyse des processus politiques dans le domaine de la promotion de la santé: comment les résultats de la recherche peuvent-ils influer sur les décisions politiques et contribuer à développer des solutions? Sur la base d'expériences positives réalisées jusqu'ici, des possibilités d'actions concrètes aux niveaux cantonal et national seront présentées en troisième partie. Les organisateurs de la conférence ont mis sur pied une offre variée avec des contributions des domaines de la recherche, de la pratique et de la politique. La table ronde de la FMH fait également partie du programme. Participez aux débats! Vous rencontrerez des scientifiques de renommée internationale comme Sir Michael Marmot, des personnalités de la politique nationale ainsi que des experts et expertes de la pratique. La conférence est organisée en commun par Santé publique Suisse (www.public-health.ch) et la Swiss School of Public Health (www.ssphplus.ch). Chaque année, elles sont soutenues par une institution académique suisse du domaine de la santé publique qui accueille la conférence et assure la direction scientifique et thématique. L'hôte de la Conférence Suisse de Santé Publique 2018, placée sous le thème "Better Health Faster», est l'Institut de droit de la santé (IDS) de l'Université de Neuchâtel (www.unine.ch/ids). La Société suisse des médecins spécialistes en prévention et santé publique (www.sgpg.ch) est responsable de la formation postgraduée et continue des médecins spécialistes en santé publique en Suisse et s'engage pour créer des conditions cadre favorables à la santé de la population. La Conférence Suisse de Santé Publique est l'une des manifestations nationales les plus importantes pour la formation continue dans ce domaine.

Nous nous réjouissons d'avance de vous rencontrer à Neuchâtel à l'occasion de la Conférence Suisse de Santé Publique 2018. 